\title{
Charge-State-Dependent Diffusion of Individual Gold Adatoms on Ionic Thin NaCl Films
}

\author{
Jascha Repp, ${ }^{1, *}$ Wolfram Steurer, ${ }^{2}$ Ivan Scivetti, ${ }^{3}$ Mats Persson, ${ }^{3}$ Leo Gross, ${ }^{2}$ and Gerhard Meyer ${ }^{2}$ \\ ${ }^{1}$ Institute of Experimental and Applied Physics, University of Regensburg, 93053 Regensburg, Germany \\ ${ }^{2}$ IBM Research-Zurich, 8803 Rüschlikon, Switzerland \\ ${ }^{3}$ Surface Science Research Centre and Department of Chemistry, University of Liverpool, Liverpool L69 3BX, United Kingdom
}

(Received 19 May 2016; published 30 September 2016)

\begin{abstract}
It is known that individual metal atoms on insulating ionic films can occur in several different (meta) stable charge states, which can be reversibly switched in a controlled fashion. Here we show that the diffusion of gold adatoms on $\mathrm{NaCl}$ thin films depends critically on their charge state. Surprisingly, the anionic species has a lower diffusion barrier than the neutral one. Furthermore, for the former we observe that the diffusion atop a bilayer of $\mathrm{NaCl}$ is strongly influenced by the interface between $\mathrm{NaCl}$ and the underlying copper substrate. This effect disappears for a trilayer of $\mathrm{NaCl}$. These observations open the prospect of controlling the diffusion properties of individual metal atoms on thin insulating films.
\end{abstract}

DOI: $10.1103 /$ PhysRevLett.117.146102

A most interesting finding is the observation that metal atoms on insulating films can exhibit several different charge states, which can be controlled on the level of individual atoms in the junction of a scanning probe microscope [1-10]. As the charge state of an adsorbate is decisive for many of its physical and chemical properties, a control of the charge state offers the prospect of controlling adsorbates in many other aspects, ranging from their spin degrees of freedom [6,7] to their catalytic activity [11]. Although the possibility of controlling the diffusion properties by means of charge-state manipulation was briefly mentioned [1], no corresponding experiments were reported so far.

Here we show from scanning tunneling microscopy (STM) experiments that the diffusion behavior of individual gold adatoms on ultrathin $\mathrm{NaCl}$ films depends strongly on its charge state and can therefore be controlled by means of STM-based manipulation [1]. Somewhat surprisingly, the negatively charged gold anions diffuse more readily as compared to the neutral ones. This result is rationalized in view of a recent analysis of the different possible adsorption sites of these adsorbates [10]. We further observe that $\mathrm{Au}$ anions on a bilayer of $\mathrm{NaCl}$ on $\mathrm{Cu}(111)$ show a diffusion pattern corresponding to the moiré pattern of the interface of $\mathrm{NaCl}$ to the underlying metal substrate. Apparently, the ionic relaxations in the polar insulating film that are present around negatively charged $\mathrm{Au}^{-}$anions $[1,12]$ interact with the bucklings of ions in bilayer $\mathrm{NaCl}$ films that arise from the incommensurate interface with the substrate.

The experiments were carried out with two homebuilt low-temperature STM apparatuses with a base temperature of $5 \mathrm{~K}$. NaCl was evaporated thermally onto a clean $\mathrm{Cu}(111)$ single-crystal surface such that defect-free (100)-terminated $\mathrm{NaCl}$ bilayer islands were formed [13] with smaller patches of a third $\mathrm{NaCl}(100)$ layer on top of them. Gold atoms were deposited with the sample inside the STM at $T<10 \mathrm{~K}$. All voltages refer to the sample bias with respect to the tip. For our diffusion study, many sequences of STM images were recorded at different temperatures. The temperature was stabilized by heating the entire STM head, being thermally only weakly coupled to the helium bath. The temperature was sensed at the STM head by means of diode thermometry. The temperature was kept stable for several hours for thermal equilibration before starting the acquisition of image sequences, from which diffusion patterns were extracted. These sequences were corrected for the remaining low creep and drift only in hindsight by means of markers provided by defects or stationary adatoms. Some sequences are available as movies; see [14]. The atomic positions were extracted numerically by locally fitting the adsorbates' protrusions in the STM images to two-dimensional Gaussian functions.

The diffusion of neutral gold adatoms $\left(\mathrm{Au}^{0}\right)$ [20] was investigated by recording several series of subsequent STM images each at a fixed temperature $T$ in the range of $50 \mathrm{~K}<T<60 \mathrm{~K}$. From each of these series, the imageto-image displacement of the observed adatom was extracted. The resulting pattern for one of these is displayed in Fig. 1(a). It reflects the square $\mathrm{NaCl}(100)$ surface lattice and is therefore compatible with the on-top $\mathrm{Cl}^{-}$adsorption site as reported earlier [1]. The image-to-image adatom displacement distribution fits the one expected for a singlestep random walk [21], indicating conventional diffusion. From each of the series of images, the hopping rate $\nu$ was extracted $[14,15]$, which plotted against the inverse temperature yields the Arrhenius plot shown in Fig. 1(b). Fitting these data to $\nu(T)=\nu_{0} \exp \left(-E_{D} / k_{B} T\right)$, where $k_{B}$ is the Boltzmann constant, yields the preexponential factor $\nu_{0}=$ $7 \times 10^{11 \pm 2} \mathrm{~s}^{-1}$ and a diffusion barrier $E_{D}=(0.156 \pm 0.02) \mathrm{eV}$.

As can be seen in Fig. 2 and the corresponding movie [14], gold anions $\left(\mathrm{Au}^{-}\right)$diffuse more readily than the $\mathrm{Au}^{0}$ 
(a) $\mathrm{Au} / \mathrm{NaCl}(2 \mathrm{ML}) / \mathrm{Cu}(111)$
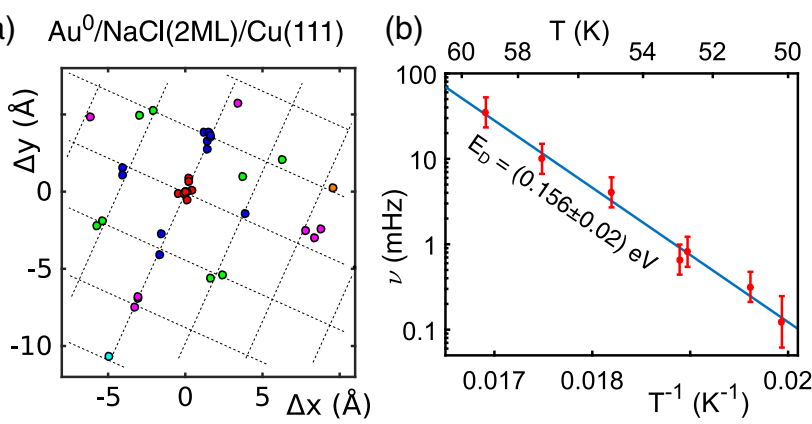

FIG. 1. (a) Image-to-image displacements of a single diffusing neutral $\mathrm{Au}^{0}$ atom adsorbed on $\mathrm{NaCl}(2 \mathrm{ML}) / \mathrm{Cu}(111)$ at $T=57.2 \mathrm{~K}$ (image-to-image time interval $\Delta t=210 \mathrm{~s}$ ). The displacements are located on a square lattice (dashed grid lines) showing that only one adsorption site per $\mathrm{NaCl}(100)$ unit cell is a stable minimum. The colors indicate the displacement distance. (b) Arrhenius plot of diffusion rate $\nu(T)$ as a function of inverse temperature $T^{-1}$. The slope of the fit line corresponds to a diffusion barrier of $0.156 \mathrm{eV}$.

adatoms. At $T \simeq 30 \mathrm{~K}, \mathrm{Au}^{-}$adatoms diffuse with hopping rates on the order of one per few minutes, whereas no hopping events are observed for $\mathrm{Au}^{0}$ adatoms consistent with the Arrhenius plot of Fig. 1(b). This enables the possibility to switch the diffusion on and off [22] by controlling the charge state. In this image sequence, two $\mathrm{Au}^{0}$ adatoms were deliberately charged (marked by red arrows), which was accompanied by an initiation of diffusion. Detailed inspection of the diffusion reveals a peculiar behavior. On the one hand, the image-to-image adatom displacements are very large compared to the $\mathrm{NaCl}$ (100) lattice spacing indicating several hops. This requires the hopping rate to be multiples of the inverse of the time delay $\Delta t$ between two subsequent images. On the other hand, however, $\mathrm{Au}^{-}$adatoms linger in certain sites for the duration of several subsequent frames, which-at first glance-is in contradiction with the above-mentioned hopping rate $[18,23]$. Furthermore, it becomes apparent that $\mathrm{Au}^{-}$adatoms tend to return to sites that were occupied previously. Given the distance of multiples of lattice spacings by which they moved in between images, it seems very unlikely that they return to the previous site just by mere coincidence. Instead, this observation suggests that certain lattice sites are energetically favored and preferentially occupied.

That behavior was analyzed in more detail, by following the diffusion of many $\mathrm{Au}^{-}$adatoms near an island edge of $\mathrm{NaCl}(2 \mathrm{ML}) / \mathrm{Cu}(111)$; see Fig. 3(a) and the corresponding movie [14]. The positions, at which these adatoms were observed were extracted and are displayed in Figs. 3(a) and 3(b). This analysis confirms the tendency of $\mathrm{Au}^{-}$ adatoms to return to previously occupied sites and a pattern of diffusion emerges that shows some regularity. This pattern is in agreement with only one adsorption site per $\mathrm{NaCl}(100)$ surface unit cell (not shown), consistent with the observation of $\mathrm{Au}^{-}$adatoms on top of $\mathrm{Cl}^{-}$atoms as reported previously [1]. The pattern of diffusion, however, is much more sparse than the surface lattice itself; that is, only a very few of all lattice sites are occupied. As only the $\mathrm{NaCl} / \mathrm{Cu}$ interface could possibly lift the degeneracy of
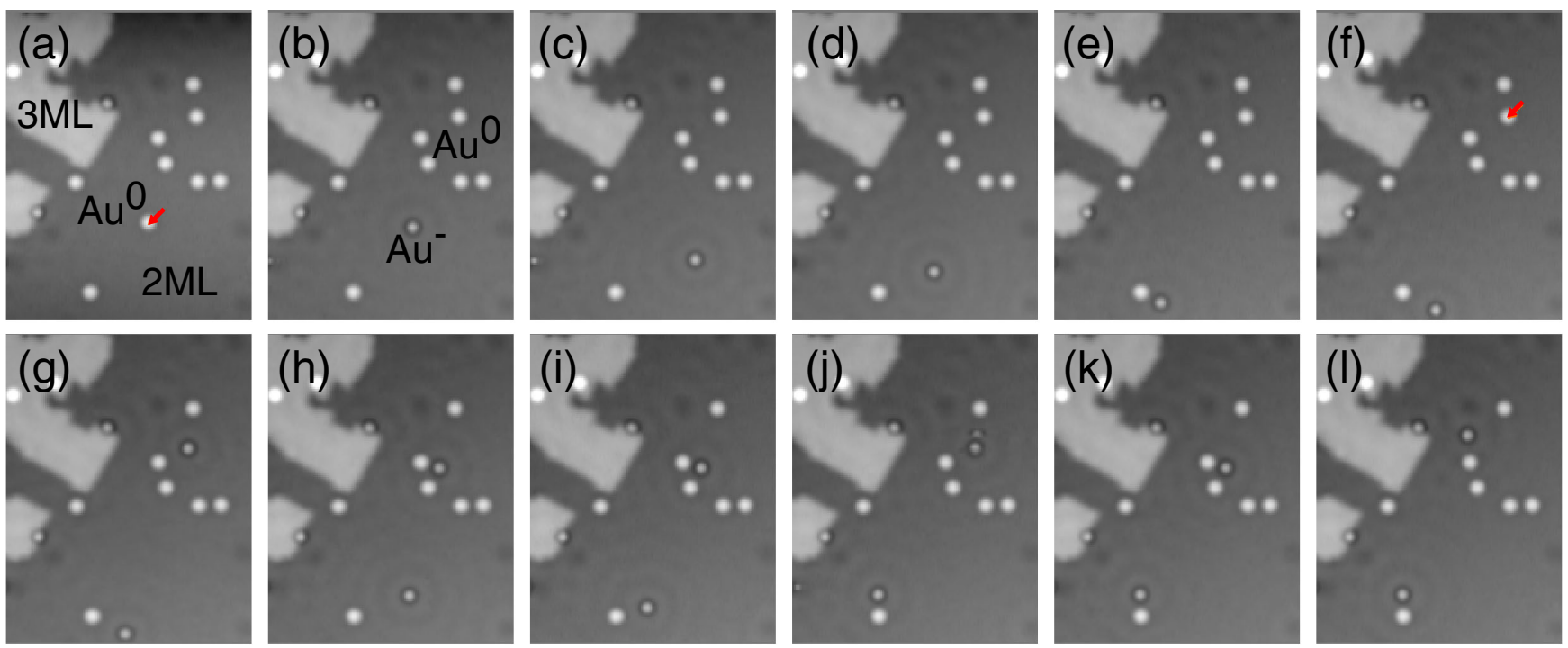

FIG. 2. STM image sequence illustrating the selective diffusion of negatively charged gold atoms $(\mathrm{Au}-)$ on $\mathrm{NaCl}(2 \mathrm{ML}) / \mathrm{Cu}(111)$ ( $T \simeq 30 \mathrm{~K} ; V=76 \mathrm{mV} ; I=0.2 \mathrm{pA} ; \Delta t=6 \mathrm{~min}$ ). (For the movie of the entire image sequence, see [14].) In frames (a) and (f), a neutral gold adatom $\left(\mathrm{Au}^{0}\right)$ was deliberately charged by means of STM manipulation to form a $\mathrm{Au}^{-}$adatom, respectively. In contrast to the $\mathrm{Au}^{0}$ adatoms, the $\mathrm{Au}^{-}$adatoms diffuse at this temperature. Although often large adatom displacements are observed between subsequent images, sometimes $\mathrm{Au}^{-}$adatoms linger in certain sites for the duration of several subsequent frames [see (h) to (i) and (j) to (1)]. Moreover, $\mathrm{Au}^{-}$adatoms tend to return to sites that were occupied previously [see frames (i)-(k) and (d)-(h); for more examples, see [14]], despite the fact that they had moved a far distance (several multiples of lattice spacings) in between images. 

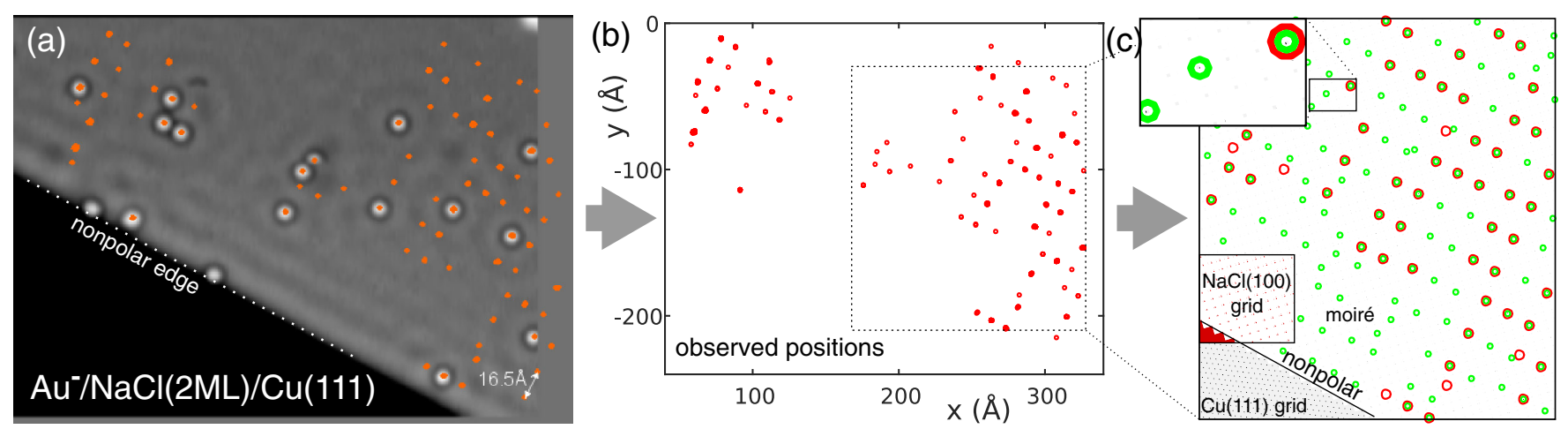

FIG. 3. Diffusion behavior of $\mathrm{Au}^{-}$adatoms on $\mathrm{NaCl}(2 \mathrm{ML}) / \mathrm{Cu}(111)$. ( $\left.T \simeq 30 \mathrm{~K} ; V=100 \mathrm{mV} ; I=0.2 \mathrm{pA} ; \Delta t=6 \mathrm{~min}\right)$. (For the movie of the entire image sequence, see [14].) The preferential occupation of a small fraction of all lattice sites becomes apparent when extracting the observed adsorbates' positions from a sequence of images. The typical spacing of these positions is much larger than the $\mathrm{NaCl}$ lattice spacing. The pattern of extracted positions displayed in (b) can be reproduced by considering the moiré pattern shown in (c), arising from overlaying the hexagonal $\mathrm{Cu}(111)$ grid with the square grid of $\mathrm{NaCl}(100)$ [see the lower left corner in (c)]. The orientation of the $\mathrm{NaCl}(100)$ is known from the observation of the nonpolar island edges as indicated in (a) and (c). Small green circles indicate the coincidence of two grid points from both lattices within $0.39 \AA$. Comparing the resulting pattern with the experimentally observed sites (larger red circles) shows an almost identical structure with all the prominent features reproduced.

otherwise equivalent lattice sites, we analyzed the moiré pattern in the observed area as is shown in Fig. 3(c). To this end, the hexagonal grid of the topmost layer of $\mathrm{Cu}(111)$, here represented as black dots in the bottom left corner of Fig. 3(c), was overlaid with the square surface lattice of $\mathrm{NaCl}(100)$. Depending on the ratio of lattice constants and the alignment angle, the moiré pattern is not necessarily periodic. Note that the ratio of the two lattice constants as well as the alignment angle are set by literature values and the observation of the strictly nonpolar island edge of $\mathrm{NaCl}$, respectively. Only within narrow bounds could these parameters be varied to reproduce the moiré pattern [24]. This pattern is depicted by the black dots representing the $\mathrm{Cu}(111)$ lattice peeping through the grid representing $\mathrm{NaCl}(100)$. As this is difficult to see for the scaling of Fig. 3(c) (see magnification in the inset), the coincidence of the $\mathrm{NaCl}$ grid with the $\mathrm{Cu}$ grid within $0.39 \AA$ [25] was marked by small green circles. The resulting moiré pattern is almost identical to the observed diffusion pattern with all the prominent features being reproduced. This analysis reveals the direct link between the diffusion and the moiré pattern and explains why less than $10 \%$ of the possible $\mathrm{NaCl}$-lattice sites are observed as being occupied by the adsorbates [26]. An influence of moire patterns to the equilibrium sites of adatoms was observed before [26]; however, site preference was noted to have a rather smooth variation. Such induced site preference by a moiré pattern can be used as a template for regularly spaced adsorbates and clusters [27-31].

Bearing the above analysis in mind, it is not surprising that large image-to-image $\mathrm{Au}^{-}$adatom displacements were observed and, at the same time, $\mathrm{Au}^{-}$adatoms could linger in one site for the duration of several subsequent frames. Turning back to Fig. 1(a), it becomes also apparent that $\mathrm{Au}^{0}$ adatoms are not affected by the moiré pattern, in stark contrast to $\mathrm{Au}^{-}$adatoms. Hence, the charging does not only affect the barrier height, but even the qualitative diffusion behavior is completely different. The selective interaction of $\mathrm{Au}^{-}$adatoms with the moiré pattern can be rationalized when considering that the charging of $\mathrm{Au}$ is accompanied by a strong relaxation pattern of the surrounding $\mathrm{NaCl}$ ions $[1,12]$. Apparently, this relaxation pattern interacts with the buckling of $\mathrm{NaCl}$ ions resulting from the interfacial moiré pattern.

Finally, the influence of the moiré pattern should diminish for thicker insulating films, as the interface is separated further from the adsorbates. We therefore also investigated the diffusion of $\mathrm{Au}^{-}$and $\mathrm{Au}^{0}$ adatoms at the same temperature of $T \simeq 30 \mathrm{~K}$ on a trilayer island of $\mathrm{NaCl}(100)$ on $\mathrm{Cu}(111)$. The result is shown in Fig. 4 and a corresponding movie [14]. As for the bilayer data at this temperature, we observe the selective diffusion of $\mathrm{Au}^{-}$adatoms, whereas $\mathrm{Au}^{0}$ adatoms stay put. In contrast to the bilayer data, however, the $\mathrm{Au}^{-}$adatoms show conventional diffusion behavior without any signs of being influenced by a moiré pattern. Instead, the diffusion patterns (see insets) show two adsorption sites per $\mathrm{NaCl}$ surface unit cell, indicating the hollow site (between two $\mathrm{Cl}^{-}$and two $\mathrm{Na}^{+}$ions) as the adsorption site in agreement with Ref. [10].

This last observation of a change in adsorption site from bi- to trilayers of $\mathrm{NaCl}$ for $\mathrm{Au}^{-}$adatoms suggests that the on-top $\mathrm{Cl}^{-}$site and the hollow site are almost degenerate in energy. Using manipulation at a temperature $T$ well below the onset of diffusion, also nonequilibrium configurations can be occupied [32]. Indeed, using this technique at $T \simeq 5 \mathrm{~K}, \mathrm{Au}^{-}$adatoms could be stabilized in both of the above-mentioned sites on the $\mathrm{NaCl}$ bilayer. This near degeneracy is in line with the observation that the diffusion barrier of $\mathrm{Au}^{-}$adatoms was lower than the one for $\mathrm{Au}^{0}$ adatoms. Relating the temperatures of the onset of diffusion 

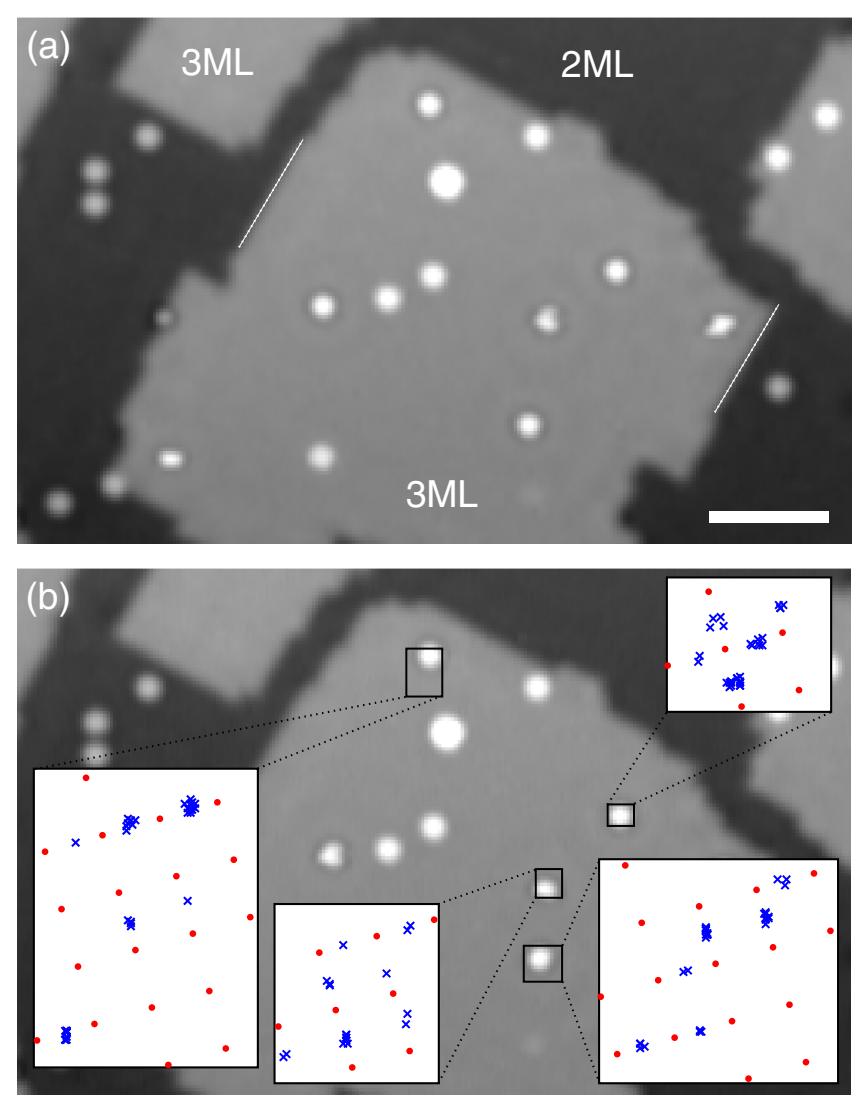

FIG. 4. STM images out of a series of $\mathrm{Au}^{0}$ and $\mathrm{Au}^{-}$adatoms on bi- (2 ML) and trilayers (3 ML) of $\mathrm{NaCl}(100)$ on $\mathrm{Cu}(111)$ ( $T \simeq 30 \mathrm{~K} ; V=150 \mathrm{mV} ; I=0.2 \mathrm{pA} ; \Delta t=6 \mathrm{~min}$ ). (For the movie of the entire image sequence, see [14].) The sequence shows also selective diffusion of $\mathrm{Au}^{-}$adatoms only. The insets in (b) show the extracted pattern of occupied positions (blue crosses) overlaid with the square $\mathrm{NaCl}$ lattice (red dots, not referring to a specific site), the orientation of which is known from the nonpolar step edges indicated in (a). In contrast to the behavior on the bilayer, $\mathrm{Au}^{-}$adatoms on trilayers of $\mathrm{NaCl}$ on $\mathrm{Cu}(111)$ do not exhibit any peculiar influence from the interfacial moiré pattern. Instead, the diffusion pattern shows two adsorption positions per square $\mathrm{NaCl}(100)$ surface unit cell, which is compatible with adsorption in a hollow site (between two $\mathrm{Cl}^{-}$ and two $\mathrm{Na}^{+}$ions). The scale bar is $5 \mathrm{~nm}$.

for $\mathrm{Au}^{-}$and $\mathrm{Au}^{0}$ adatoms with the diffusion barrier of $\mathrm{Au}^{0}$ adatoms extracted from the Arrhenius plot, we estimate the diffusion barrier of $\mathrm{Au}^{-}$adatoms to be $E_{B} \simeq$ $(0.09 \pm 0.02) \mathrm{eV}$ on the $\mathrm{NaCl}$ bi- and trilayer [14].

The observation that the diffusion barrier of $\mathrm{Au}^{-}$ adatoms on the $\mathrm{NaCl}$ trilayer is relatively small and lower than the one for $\mathrm{Au}^{0}$ adatoms is corroborated by density functional theory calculations based on a perfect conductor model of the metal substrate $[33,34]$. The calculations were carried out using an implementation of this model [33] in VASP [35]. The electron-ion-core interactions were handled by the projector augmented wave method (PAW) [36], and the exchange-correlation energy was treated using the version optB86b [37] of the van der Waals density functional [38-40]. Each layer contained $16 \mathrm{Na}$ and 16 $\mathrm{Cl}$ ions [41]. Since the measured relative change of the $\mathrm{NaCl}$ bilayer surface lattice constant $a$ from the truncated bulk surface lattice constant $a_{B}$ is very close to the corresponding calculated relative change of $\left(a-a_{B}\right) / a_{B}=$ $-1.9 \%$ of the freestanding bilayer, the calculated surface lattice constant of the freestanding trilayer was used for the trilayer corresponding to $\left(a-a_{B}\right) / a_{B}=-1.4 \%$ [42]. The most stable adsorption sites for the $\mathrm{Au}^{0}$ and $\mathrm{Au}^{-}$adatoms were found to be the top and hollow sites, respectively, in agreement with experiments. Using nudged elastic band calculations [43], the lowest potential energy barrier of $79 \mathrm{meV}$ for the diffusion of the $\mathrm{Au}^{-}$adatoms was found to be between nearest neighboring hollow sites [44]. The transition state was found to be slightly displaced from the fourfold symmetric top site. In agreement with the experiments, this barrier is smaller than the calculated potential energy barrier of $96 \mathrm{meV}$ for $\mathrm{Au}^{0}$ adatoms between nearest neighboring top sites.

In conclusion, we found that $\mathrm{Au}^{0}$ and $\mathrm{Au}^{-}$adatoms exhibit fundamentally different diffusion properties. The diffusion barrier of the anion is only roughly $60 \%$ of that of the neutral species. The diffusion of $\mathrm{Au}^{-}$adatoms on a bilayer is strongly affected by the interfacial $\mathrm{NaCl}(100)$ / $\mathrm{Cu}(111)$ moiré pattern, whereas the diffusion of $\mathrm{Au}^{0}$ atoms is not. As an interesting perspective, these observations suggest that the diffusion of anions on top of ultrathin films can be guided by designing suitable moiré patterns from the choice of the interface [27-31]. The mechanisms behind the peculiar diffusion behavior reported here are not limited to the system studied and therefore will have implications for the wide range of adsorbate-substrate systems that show charge bistability [1-10,45-47].

We thank Rolf Allenspach for helpful comments. Financial support by the Volkswagen Foundation (Lichtenberg program), the European Research Council (Advanced Grant "CEMAS"), the Leverhulme Trust (F/00 025/AQ), and the allocations of computer resources at Chadwick, The University of Liverpool are gratefully acknowledged.

*jascha.repp@ur.de

[1] J. Repp, G. Meyer, F. E. Olsson, and M. Persson, Science 305, 493 (2004).

[2] G. Pacchioni, L. Giordano, and M. Baistrocchi, Phys. Rev. Lett. 94, 226104 (2005).

[3] F. E. Olsson, S. Paavilainen, M. Persson, J. Repp, and G. Meyer, Phys. Rev. Lett. 98, 176803 (2007).

[4] M. Sterrer, T. Risse, U. Martinez Pozzoni, L. Giordano, M. Heyde, H.-P. Rust, G. Pacchioni, and H.-J. Freund, Phys. Rev. Lett. 98, 096107 (2007).

[5] L. Giordano, G. Pacchioni, J. Goniakowski, N. Nilius, E. D. L. Rienks, and H.-J. Freund, Phys. Rev. Lett. 101, 026102 (2008). 
[6] J. Park, B. D. Yu, and H. Kim, Phys. Rev. B 79, 233407 (2009).

[7] Y.-S. Fu, T. Zhang, S.-H. Ji, X. Chen, X.-C. Ma, J.-F. Jia, and Q.-K. Xue, Phys. Rev. Lett. 103, 257202 (2009).

[8] L. Gross, F. Mohn, P. Liljeroth, J. Repp, F. J. Giessibl, and G. Meyer, Science 324, 1428 (2009).

[9] B. Huang, H. Xiang, J. Yu, and S.-H. Wei, Phys. Rev. Lett. 108, 206802 (2012).

[10] W. Steurer, J. Repp, L. Gross, I. Scivetti, M. Persson, and G. Meyer, Phys. Rev. Lett. 114, 036801 (2015).

[11] B. Yoon, H. Häkkinen, U. Landman, A. S. Wörz, J.-M. Antonietti, S. Abbet, K. Judai, and U. Heiz, Science 307, 403 (2005).

[12] J. Goniakowski, C. Noguera, L. Giordano, and G. Pacchioni, Phys. Rev. B 80, 125403 (2009).

[13] R. Bennewitz, V. Barwich, M. Bammerlin, C. Loppacher, M. Guggisberg, A. Baratoff, E. Meyer, and H.-J. Güntherodt, Surf. Sci. 438, 289 (1999).

[14] See Supplemental Material at http://link.aps.org/ supplemental/10.1103/PhysRevLett.117.146102for the analysis of the temperature-dependent diffusion and the movies created from STM image sequences, which includes Refs. [15-19].

[15] G. Kellogg, Surf. Sci. Rep. 21, 1 (1994).

[16] B. S. Swartzentruber, Phys. Rev. Lett. 76, 459 (1996).

[17] J. D. Wrigley, M. E. Twigg, and G. Ehrlich, J. Chem. Phys. 93, 2885 (1990).

[18] D. C. Senft and G. Ehrlich, Phys. Rev. Lett. 74, 294 (1995).

[19] H. Ibach, Physics of Surfaces and Interfaces (SpringerVerlag, Berlin, 2006).

[20] M. H. Hakala, O. H. Pakarinen, and A. S. Foster, Phys. Rev. B 78, 045418 (2008).

[21] T. R. Linderoth, S. Horch, E. Lægsgaard, I. Stensgaard, and F. Besenbacher, Phys. Rev. Lett. 78, 4978 (1997).

[22] R. Otero, F. Hummelink, F. Sato, S. B. Legoas, P. Thostrup, E. Laegsgaard, I. Stensgaard, D. S. Galvao, and F. Besenbacher, Nat. Mater. 3, 779 (2004).

[23] M. Schunack, T. R. Linderoth, F. Rosei, E. Lægsgaard, I. Stensgaard, and F. Besenbacher, Phys. Rev. Lett. 88, 156102 (2002).

[24] $\mathrm{A} \mathrm{NaCl} / \mathrm{Cu}$ lattice-constant ratio of 1.532 and a relative alignment angle between the two grids of $22.25^{\circ}$ (nonpolar versus close-packed directions) were obtained as best fit parameters.

[25] The distance of $0.39 \AA$ was arbitrarily chosen to fit the observed pattern.
[26] N. Nilius, E. D. L. Rienks, H.-P. Rust, and H.-J. Freund, Phys. Rev. Lett. 95, 066101 (2005).

[27] A. T. N'Diaye, S. Bleikamp, P. J. Feibelman, and T. Michely, Phys. Rev. Lett. 97, 215501 (2006).

[28] J. Mao, H. Zhang, Y. Jiang, Y. Pan, M. Gao, W. Xiao, and H.-J. Gao, J. Am. Chem. Soc. 131, 14136 (2009).

[29] J. Lu, P. S. E. Yeo, Y. Zheng, Z. Yang, Q. Bao, C. K. Gan, and K. P. Loh, ACS Nano 6, 944 (2012).

[30] K. Wu, H. Zhang, Y. Wang, Y. Lu, Y. Cai, J. Song, H. Li, S. Bao, and P. He, Nanotechnology 24, 215302 (2013).

[31] Y. Han and J. W. Evans, J. Chem. Phys. 143, 164706 (2015).

[32] J. Repp, G. Meyer, K.-H. Rieder, and P. Hyldgaard, Phys. Rev. Lett. 91, 206102 (2003).

[33] I. Scivetti and M. Persson, J. Phys. Condens. Matter 25, 355006 (2013).

[34] I. Scivetti and M. Persson, J. Phys. Condens. Matter 26, 135003 (2014).

[35] G. Kresse and D. Joubert, Phys. Rev. B 59, 1758 (1999).

[36] P. E. Blöchl, Phys. Rev. B 50, 17953 (1994).

[37] J. Klimeš, D. R. Bowler, and A. Michaelides, Phys. Rev. B 83, 195131 (2011).

[38] M. Dion, H. Rydberg, E. Schröder, D. C. Langreth, and B. I. Lundqvist, Phys. Rev. Lett. 92, 246401 (2004).

[39] T. Thonhauser, V. R. Cooper, S. Li, A. Puzder, P. Hyldgaard, and D. C. Langreth, Phys. Rev. B 76, 125112 (2007).

[40] G. Román-Pérez and J. M. Soler, Phys. Rev. Lett. 103, 096102 (2009).

[41] The PAW potential for the Na atom included the $p$ semicore. The plane wave energy cutoff was $400 \mathrm{eV}$. The surface Brillouin zone was sampled using a $2 \times 2 k$ point grid.

[42] The experimental literature value of $a_{B}$ and the value calculated in this work are 3.988 and $3.971 \AA$, respectively.

[43] G. Mills, H. Jónsson, and G. K. Schenter, Surf. Sci. 324, 305 (1995).

[44] This value includes repulsive dipole-dipole corrections between periodic images. This correction was confirmed for hollow and top sites using a larger surface unit cell with $36 \mathrm{Na}$ and $36 \mathrm{Cl}$ ions in each layer.

[45] S. W. Wu, N. Ogawa, and W. Ho, Science 312, 1362 (2006).

[46] S. W. Wu, N. Ogawa, G. V. Nazin, and W. Ho, J. Phys. Chem. C 112, 5241 (2008).

[47] I. Swart, T. Sonnleitner, and J. Repp, Nano Lett. 11, 1580 (2011). 